\title{
ARTICLE
}

\section{Mutual separation of trivalent lanthanide and actinides by hydrophilic and lipophilic multidentate diamides}

\author{
Yuji Sasaki ${ }^{*}$ and Keisuke Morita \\ Japan Atomic Energy Agency, 2-4 Shirakata, Tokai-mura, Naka-gun, Ibaraki-ken, 319-1195, Japan
}

\begin{abstract}
Not only the separation of actinide (An) from lanthanide (Ln) but also the separation of Am and $\mathrm{Cm}$ are required. Although there includes difficulty of the mutual separation of these metals, we take the solvent extraction method using lipophilic and hydrophilic diamides and aminopolyacetic acid dissolved in both aqueous and organic phases, in order to get high separation factors. It is clear that combination of two ligands (TODGA as an extractant and DTPA or DTPAbisamide as a masking agent) give around and higher 10 of SF for $\mathrm{Nd} / \mathrm{Am}$ and $\mathrm{Nd} / \mathrm{Cm}$, and combination of two ligands (DGA and DOODA ligands) give around 2 of $\mathrm{SF}$ for $\mathrm{Am} / \mathrm{Cm}$ in column separation.
\end{abstract}

Keywords: actinides; lanthanides; separation; solvent extraction; extraction chromatography

\section{Introduction}

Actinide (An) separation from lanthanide (Ln) is required for the purpose of removal of matrix lanthanides, reduction of neutron scavenging nuclides, and reduction of strong radioactivities and heats. In addition, it is also important to separate $\mathrm{Am}$ from $\mathrm{Cm}$, the reasons why are the decrease of the heat source from MA fuel and reduction of the radioactive exposure by the neutrons that $\mathrm{Cm}-244$ emit owing to the spontaneous fission. However, it is well-known that the mutual separation of these metals is quite difficult because these metal ions show the same oxidation state, similar ionic radii, and adjacent in the periodic table. Recently, the extractants, DGA and DOODA, well-extract An and Ln, are developed and used to see the many properties[1-4]. In this work, we take the solvent extraction and extraction chromatography using lipophilic and hydrophilic diamides and aminopolyacetic acid dissolved in both aqueous and organic phases, in order to get high mutual separation factors.

\section{Experimental}

Two $\mathrm{mL}$ of the organic phase of extractants (TODGA $\left(N, N, N^{*}, N^{*}\right.$-tetraoctyl-diglycolamide) or DOODA(C8) $\left(N, N, N^{\prime}, N^{\prime}\right.$-tetraoctyl-dioxaoctanediamide) $) / n$-dodecane $[1,2]$ is mixed and shaken with the same volume of the aqueous phase, which contains metal ion with and without masking agent (water-soluble DGA, DOODA, EDTA (ethylenediamine- $N, N, N^{\prime}, N^{\prime}$-tetraacetic acid), EDTAbisamide (ethylenediamine- $N, N^{*}$-diacetic

*Corresponding author. Email: sasaki.yuji@jaea.go.jp bisamicacid), DTPA (Diethylenetriamine- $N, N, N N^{\prime}, N^{\prime \prime}, N$ “" pentaacetic acid) and DTPAbisamide (Diethylenetriamine$N, N, N$ " $N$ " " $N$ " "-triacetic bisamicacid)) [5-7] in $\mathrm{HNO}_{3}$ or $\mathrm{NaNO}_{3}$, at $25{ }^{\circ} \mathrm{C}$ for $30 \mathrm{~min}$. After centrifugation, the radioactivity of the aqueous and the organic phases is measured by a liquid scintillation counter. The non-radioactive metal concentrations in the aqueous phases were measured by ICP-AES or ICP-MS, and the concentrations of the organic phase are obtained by subtraction of aqueous phase concentration from initial one. Here, the concentration of $\mathrm{Ln}$ and An are 10-100 ppm and sub-ppb level for solvent extraction and the experimental errors are considered to be within $5 \%$. The $D(\mathrm{M})$ values are defined as the ratio of $[\mathrm{M}]$ in the organic phase versus $[\mathrm{M}]$ in the aqueous phase. [M] means the metal concentration in each phase.

The chromatographic experiments are performed as follows: the extractants were impregnated in $\mathrm{SiO}_{2}-\mathrm{P}$. The solid absorbent is packed in the column size of 6 $\mathrm{mm} \varphi \mathrm{x} 150 \mathrm{~mm} \mathrm{H}$. The dead volume is evaluated to $3 \mathrm{ml}$. The desired solution is poured in this column as the flow rate of 2.5-3.3 $\mathrm{ml} / \mathrm{min}$. After elution, each metal in eluent is measured by the suitable instruments.

\section{Results and discussion}

\subsection{Separation of $A n / L n$ by EDTA and EDTAbisamide}

We use the extraction of TODGA in the organic phase and EDTA or EDTAbisamide in the aqueous phase. One of the advantages to use EDTAbisamide is to have high solubility in water. In Figure 1, Ln patterns $(D(\mathrm{Ln})$ vs. Atomic number of $\mathrm{Ln})$ obtained are shown at 

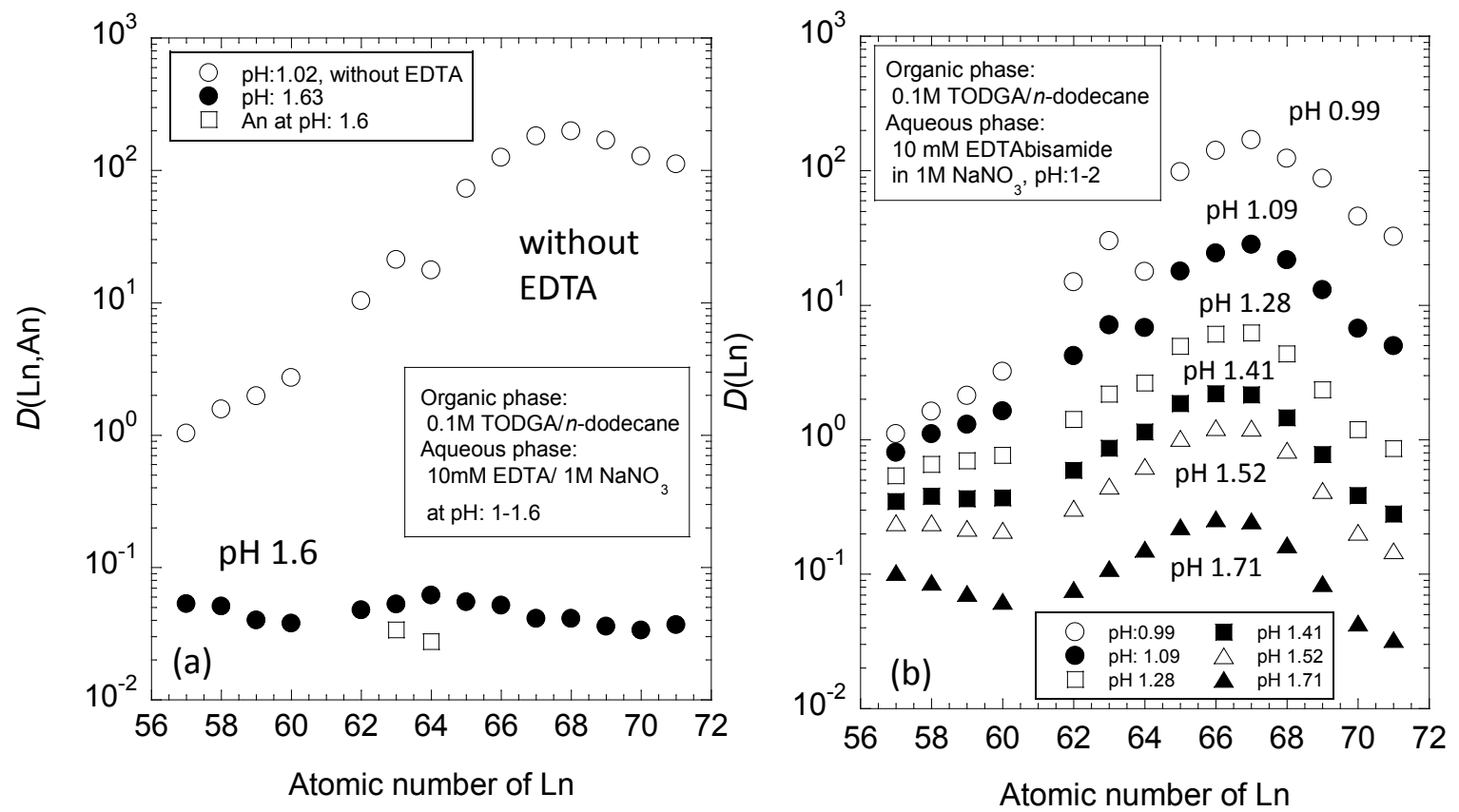

Figure 1. Ln pattern using 0.1M TODGA $n$-dodecane and EDTA or EDTAbisamide/ $1 \mathrm{M} \mathrm{NaNO}$ - with the different pH, (a): EDTA, (b): EDTAbisamide.

different $\mathrm{pH}$ condition. Figure 1(a) indicates the result of EDTA and Figure 1(b) is for EDTAbisamide. These compounds have carboxylic acid groups and are dissociated in solutions, the carboxylic acid groups may react with $\mathrm{Ln}$ and $\mathrm{An}$ at high $\mathrm{pH}$ conditions. As shown in Figures 1(a) and (b), the gradual decrease of $D$ against $\mathrm{pH}$ suggest the complexation of Ln with EDTA and EDTAbisamide in the aqueous phase. It seems that the decrease of $D$ for heavy $\mathrm{Ln}$ is more remarkable than light Ln, indicating that the complexation with heavy Ln is greater than light $\mathrm{Ln}$. At high $\mathrm{pH}$, Ln pattern show the almost flat shapes and less deviation among $14 \mathrm{Ln}$ metal ions, which indicates that mutual separation of $\mathrm{Ln}$ is quite difficult at such condition. The separation of An and Ln can be evaluated using the results in Figure 1(a) for EDTA and Figure 1(b) for EDTAbisamide. From Figure 1(a), small difference between $D(\operatorname{Ln})$ and $D(\mathrm{An})$ is observed, which suggests EDTA works hardly mutual separation of Ln and An.

Figure 2 shows the results of SF (separation factor) of $\mathrm{Ln} / \mathrm{An}$ against $\mathrm{pH}$ using EDTAbisamide. From Figure 2, $\mathrm{SF}$ of $\mathrm{Ln} / \mathrm{An}$ by EDTAbisamide is changeable at different $\mathrm{pH}$, SF seems high at high $\mathrm{pH}$ condition. Probably, the different chemical reaction of La from those of Am and Cm occurs.

\subsection{Separation of An/Ln by DTPA and DTPAbisamide}

We use the extraction system of TODGA in the organic phase and DTPA or DTPAbisamide in the aqueous phase. One of the advantages to use DTPAbisamide is to have high solubility in water. In Figure 3, Ln patterns are shown at different $\mathrm{pH}$ condition. Figure 3(a) indicates the result of DTPA and Figure 3(b) is for DTPAbisamide, all data in Figure 3(a)

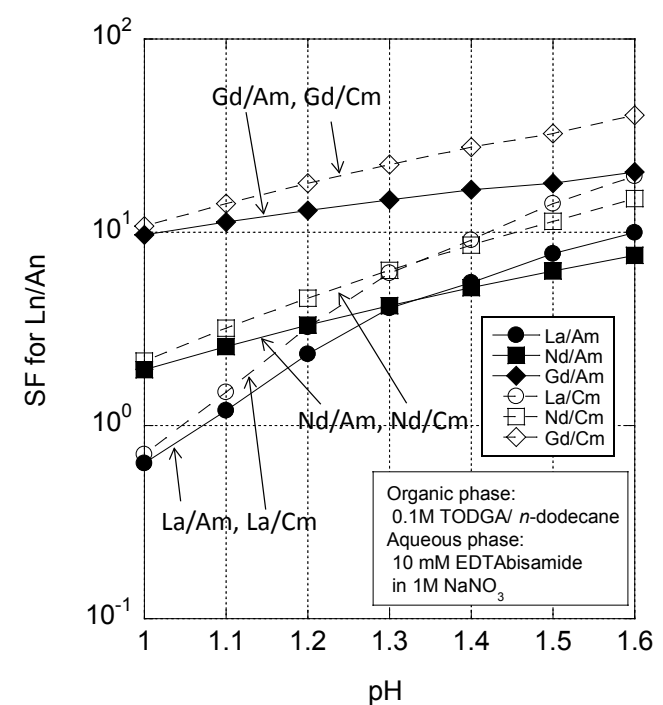

Figure 2. Separation factors obtained from the results in Figure 1(b).

are refered from reference [7]. Both figures indicate that $D$ values of middle and heavy $\mathrm{Ln}$ change widely compared to light $\mathrm{Ln}$ when $\mathrm{pH}$ increases from 1 to 2 , which means these water-soluble reagents react strongly to middle and heavy Ln. At the same $\mathrm{pH}$ condition, $D(\mathrm{Ln})$ using DTPAbisamide is 10 times lower than that using DTPA. Compared to EDTA and EDTAbisamide, it is clear that DTPA and DTPAbisamide indicate the strong complexation with middle and heavy Ln.

Using these $D$ values, $\mathrm{SF}$ for $\mathrm{Ln} / \mathrm{An}$ is studied. Figure 4 show SF against $\mathrm{pH}$ values, Figure 4(a) is for DTPA and Figure 4(b) is for DTPAbisamide. From Figure 4, almost constant or gradual decrease of SF for 

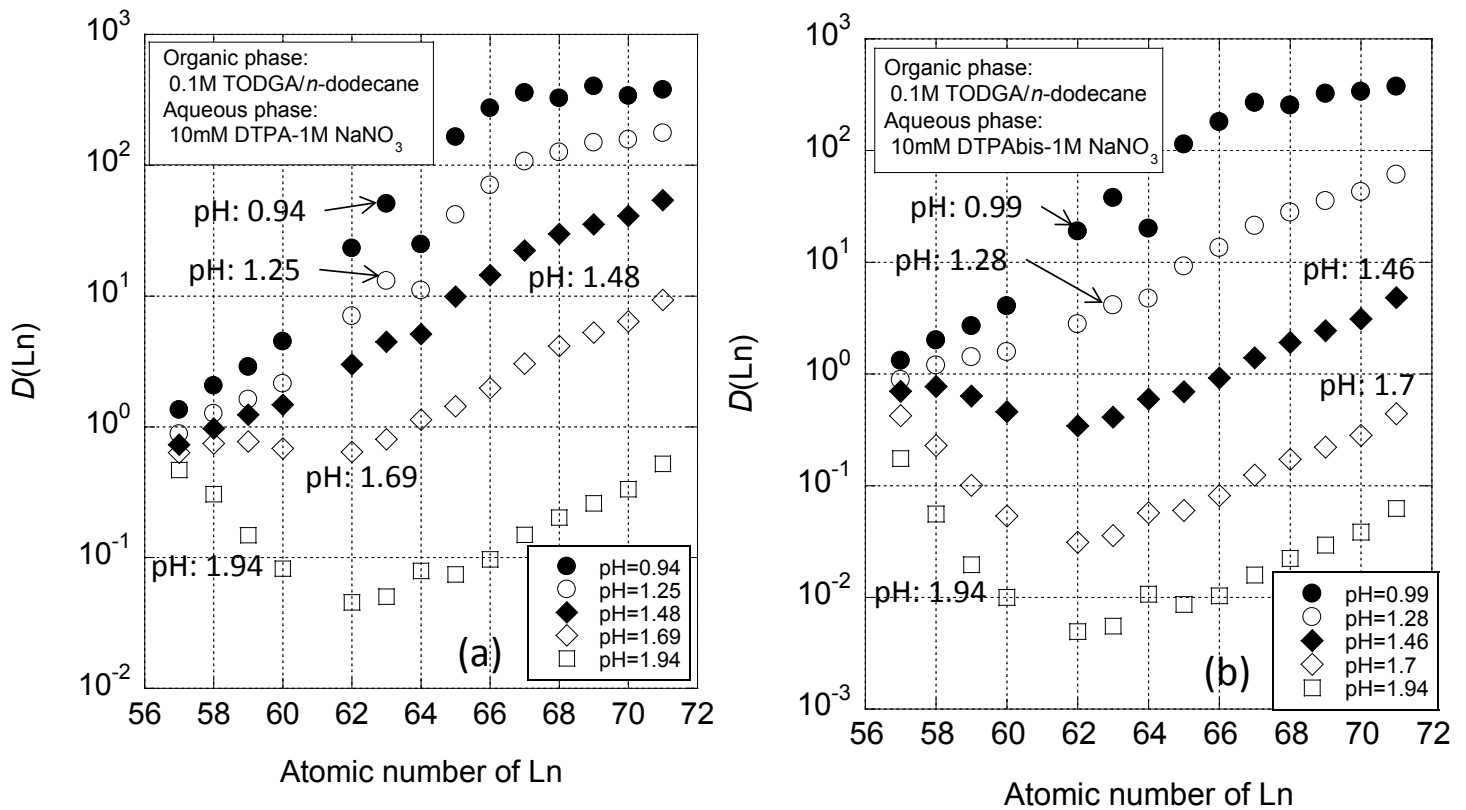

Figure 3. Ln pattern using 0.1M TODGA/n-dodecane and DTPA* or DTPAbisamide/ $1 \mathrm{M} \mathrm{NaNO}_{3}$ with the different $\mathrm{pH}$, (a): DTPA, (b): DTPAbisamide.

*: Data is referred by the Ref. [7].
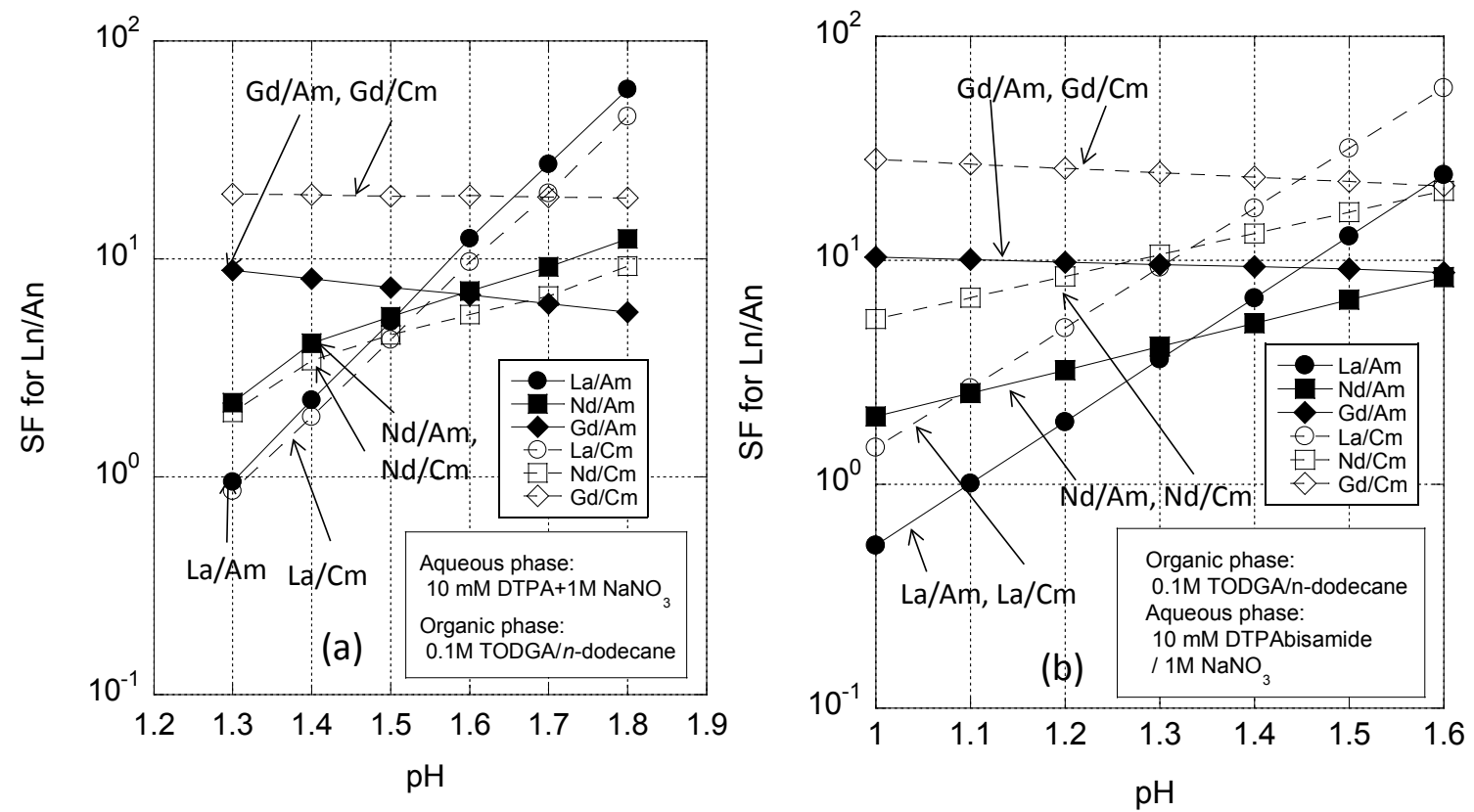

Figure 4. Separation factors obtained from the results in Figure 3, *: Data is referred by the reference [7].

$\mathrm{Gd} / \mathrm{An}$ with $\mathrm{pH}$ is observed, which indicates that $\mathrm{Gd}$ and $\mathrm{Am}$ or $\mathrm{Cm}$ show the same chemical reaction at the $\mathrm{pH}$ conditions. However, SF for $\mathrm{La} / \mathrm{An}$ and $\mathrm{Nd} / \mathrm{An}$ increase clearly with $\mathrm{pH}$, which suggests different chemical reaction between these metal ions. This behavior can be seen in solvent extraction with EDTAbisamide (Figure 2). La and $\mathrm{Nd}$ have the different reactions with dissociated DTPA or DTPAbisamide from those of Am and $\mathrm{Cm}$, then changeable SF values are observed. At (a): DTPA*, (b): DTPAbisamide

$\mathrm{pH}$ 1.6-1.8, it appears that $\mathrm{SF}$ for $\mathrm{Nd} / \mathrm{Am}$ and $\mathrm{Nd} / \mathrm{Cm}$ are approximate 10 or over.

\subsection{Separation of $\mathrm{Am} / \mathrm{Cm}$}

In order to obtain high separation factor of Am and $\mathrm{Cm}$, both of extractant and masking agent are used in an extraction system. In this work, we take Ln pattern as criterion how to decide two reagents. For example, Ln 
patterns for extractants of DGA and DOODA are shown in Figure 5. As shown in Figure 5, DGA shows preference of heavy Ln and DOODA shows in reverse. Trivalent An is similar chemical behavior as Ln, then the combination of extractant of DGA in organic phase and masking agent of water-soluble DOODA or extractant of DOODA and water-soluble DGA may show the effective separation.

We take the preliminary test of extraction chromatography in order to see the mutual separation of Ln and An. The results are shown in Figures 6 and 7, and summary of column separation are shown in Table 1. Figure 6 indicates the results of elution of Ln using eluent of water-soluble DOODA through TODGA column (Figure 6(a)) and that using water-soluble DGA through DOODA column (Figure 6(b)). Figure 6(a) shows the elution trend from light to heavy $\mathrm{Ln}$, because TODGA in absorbent shows the strong affinity with heavy Ln. On the other hand, Figure 6(b) indicates the elution trend from heavy to light ones, which corresponds to the affinity trend of DOODA in absorbent. In these figures, easy separation between $\mathrm{Nd}$ and $\mathrm{Sm}$ is found.

Am and $\mathrm{Cm}$ show the most similar behavior to these metals in $\mathrm{Ln}$ series. The results of $\mathrm{Am}$ and $\mathrm{Cm}$ are shown in Figure 7. From this figure, Am and $\mathrm{Cm}$ can be separated by these extraction columns. Table 1 shows the results of these column separation, namely $K_{\mathrm{d}}$ values, peak position, half width, recovery and $D$ values by solvent extraction as a reference. Using these values, SF for column separation of $\mathrm{Am} / \mathrm{Cm}$ are 2.25 (DGA column) and 1.95 (DOODA column). These results are satisfied for mutual separation of MA.

\section{Conclusion}

The separation of not only Ln and An but also Am

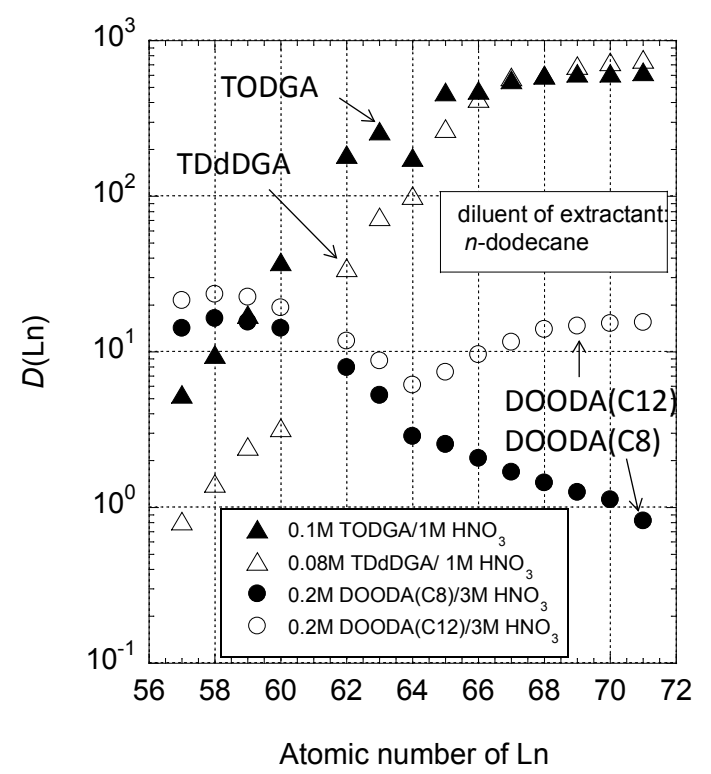

Figure 5. Ln patterns for extractants of TODGA, TDdDGA, $\operatorname{DOODA}(\mathrm{C} 8)$ and DOODA(C12)

and $\mathrm{Cm}$ using solvent extraction and extraction chromatography are studied. Utility of two ligands (one is extractant, another is masking agent) is very important to get high separation factors between these metal ions, in this work we employ Ln pattern as criterion in order to decide the available ligands for this purpose. It is clear that combination of two ligands (TODGA as an extractant and DTPA or DTPAbisamide as a masking agent) give around and higher 10 of $\mathrm{SF}$ for $\mathrm{Nd} / \mathrm{Am}$ and $\mathrm{Nd} / \mathrm{Cm}$, and combination of two ligands (DGA and DOODA ligands) give around 2 of $\mathrm{SF}$ for $\mathrm{Am} / \mathrm{Cm}$ in column separation.
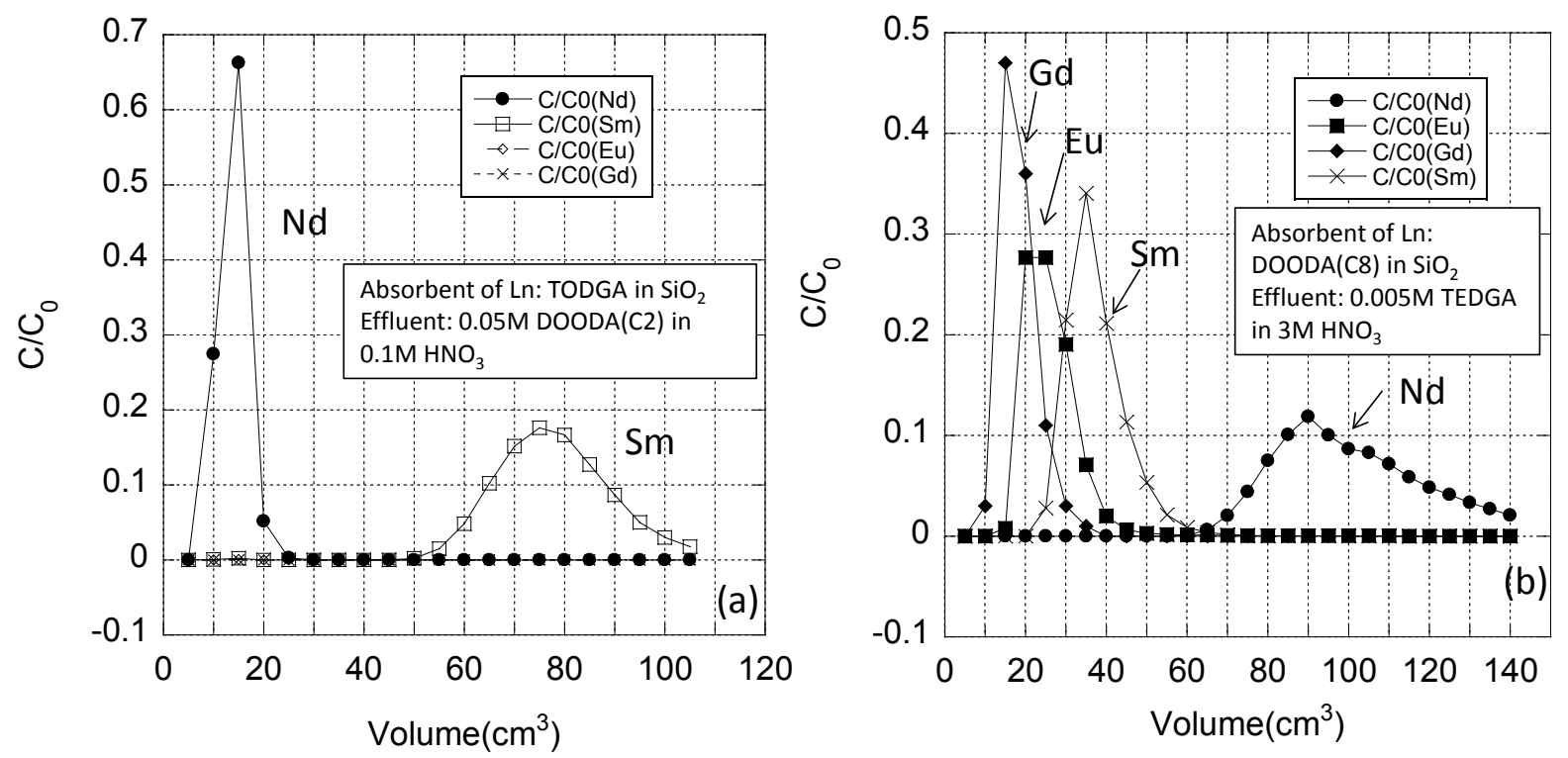

Figure 6. Column separation of Ln using DGA and DOODA column, (a): DGA column, (b): DOODA column. 

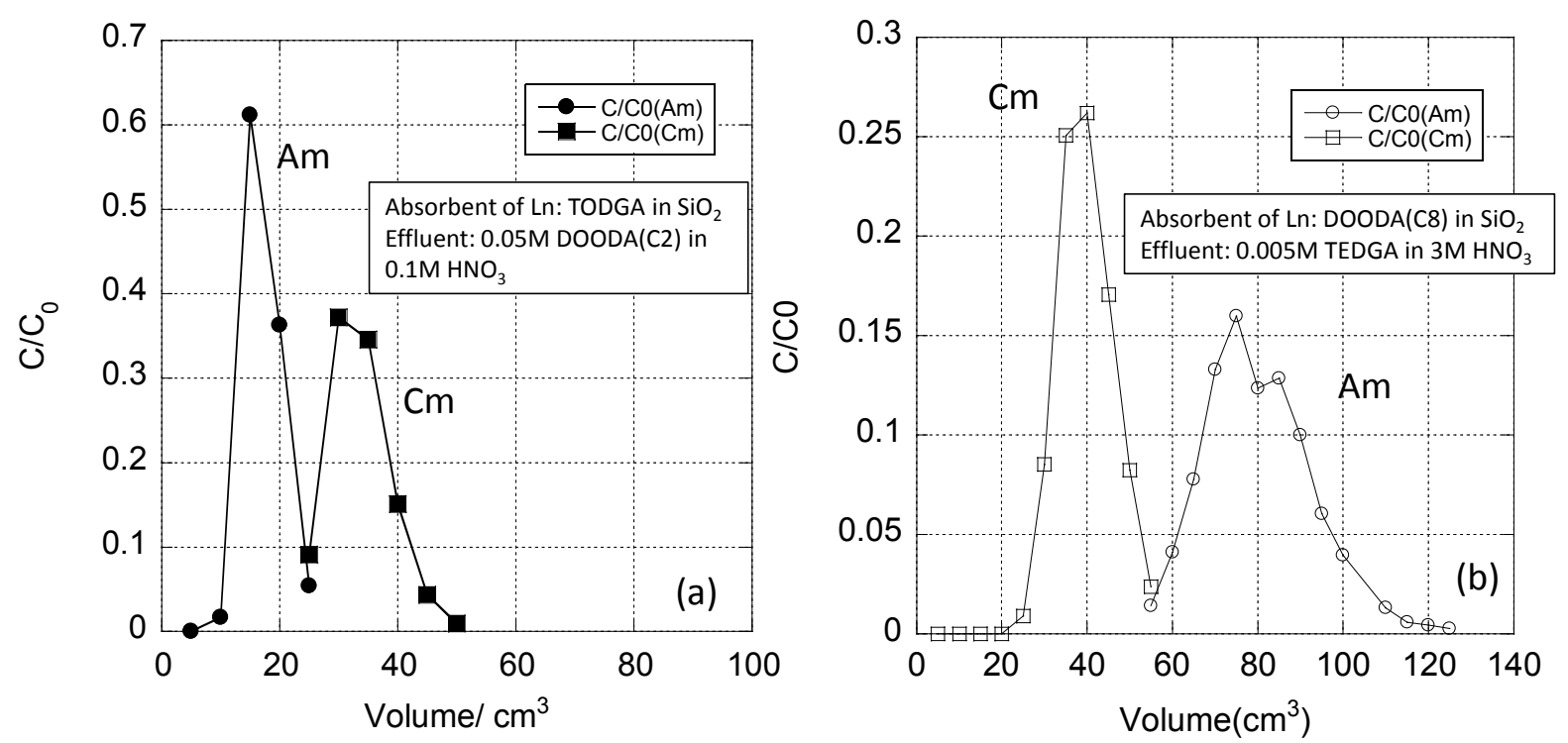

Figure 7. Column separation of Am and Cm using DGA and DOODA column, (a): DGA column, (b): DOODA column

Table 1. Results of column separation of An and Ln.

1. TODGA column (effluent: $0.05 \mathrm{M} \mathrm{DOODA}(\mathrm{C} 2) / 0.1 \mathrm{MHNO}_{3}$ )

\begin{tabular}{lccccc}
\hline & $K d$ & Peak position & Half width recovery & $\begin{array}{l}D \text { by solvent extraction } \\
\left(0.1 \mathrm{M} \mathrm{TODGA} / 0.1 \mathrm{MNO}_{3}\right)\end{array}$ \\
\hline $\mathrm{Nd}$ & 1.61 & $15 \mathrm{ml}$ & $8 \mathrm{ml}$ & $99 \%$ & 0.012 \\
$\mathrm{Sm}$ & 24.1 & 75 & 25 & 98 & \\
$\mathrm{Eu}$ & 71.9 & - & - & 0 & 0.0363 \\
$\mathrm{Gd}$ & 163 & - & - & 0 & \\
$\mathrm{Am}$ & 1.51 & 15 & 10 & 105 & 0.015 \\
$\mathrm{Cm}$ & 3.33 & 30 & 15 & 101 & 0.0365 \\
\hline
\end{tabular}

2. DOODA column (effluent: $0.005 \mathrm{M}$ TEDGA/3MHNO${ }_{3}$ )

\begin{tabular}{llllcc}
\hline & $K d$ & Peak position & Half width & Recovery & $\begin{array}{l}D \text { by solvent extraction } \\
\left(0.3 \mathrm{M} \text { DOODA/ } 3 \mathrm{MNO}_{3}\right)\end{array}$ \\
\hline $\mathrm{Nd}$ & 70.45 & $90 \mathrm{ml}$ & $35 \mathrm{ml}$ & $94 \%$ & 21 \\
$\mathrm{Sm}$ & 25.57 & 35 & 15 & 100 & 12.05 \\
$\mathrm{Eu}$ & 14.26 & 22.5 & 15 & 86 & 8.97 \\
$\mathrm{Gd}$ & 8.45 & 15 & 10 & 104 & 6.15 \\
$\mathrm{Am}$ & 47.0 & 75 & 30 & 90 & 13.3 \\
$\mathrm{Cm}$ & 17.7 & 40 & 20 & 88 & 8.5 \\
\hline
\end{tabular}

\section{Acknowledgements}

The authors would like to thank to Mr. M. Ito and Mr.

Y. Nakahara for the useful comments and experiments.

\section{References}

[1] Y. Sasaki, Y. Sugo, S. Suzuki and S. Tachimori,
The novel extractants, diglycolamides, for the extraction of lanthanides and actinides in $\mathrm{HNO}_{3}$-n-dodecane system, Solvent Extr. Ion Exch. 19 (2001), pp.91-103.

[2] Y. Sasaki, Y. Morita, Y. Kitatsuji and T. Kimura, Extraction behavior of actinides and metal ions by the promising extractant, $N, N, N^{\prime}, N^{\prime}$-tetraoctyl-3, 
6-dioxaoctanediamide (DOODA), Solvent Extr. Ion Exch. 28 (2010), pp.335-349.

[3] S.A. Ansari, P. Pathak, P.K. Mohapatra and V.K. Manchanda, Chemistry of diglycolamides: Promising extractants for actinide partitioning, Chem. Rev. 112 (2012), pp.1751-1772.

[4] A. Leoncini, J. Huskens and W. Verboom, Ligands for f-element extraction used in nuclear fuel cycle, Chem. Soc. Rev. 46 (2017), pp.7229-7273.

[5] Y. Sasaki, Y. Sugo, Y. Kitatsuji, A. Kirishima, T. Kimura and G.R. Choppin, Complexation of various metals by water-soluble diglycolamide
(DGA), Anal. Sci. 23 (2007), pp.727-731.

[6] Y. Sasaki, Y. Kitatsuji, Y. Tsubata, Y. Sugo and Y. Morita, Separation of Am, Cm and Ln by the solvent extraction with hydrophilic and lipophilic organic ligands, Solvent Extr. Res. Dev. Jpn. 18 (2011), pp.93-101.

[7] A. Apichaibukol, Y. Sasaki and Y. Morita, Effect of DTPA on the extractions of actinides(III) and lanthanides(III) from nitrate solution into TODGA/n-dodecane, Solvent Extr. Ion Exch. 22 (2004), pp.997-1011. 